\title{
Rapid Identification of Biallelic SPTB Mutation in a Neonate with Severe Congenital Hemolytic Anemia and Liver Failure
}

\author{
Christopher M. Richmond ${ }^{\mathrm{a}, \mathrm{b}}$ Sally Campbell ${ }^{\mathrm{c}}$ Hee W. Foo ${ }^{\mathrm{d}}$ \\ Sebastian Lunke ${ }^{a, e, f}$ Zornitza Stark ${ }^{a, f}, g$ Amanda Moody ${ }^{h}$ \\ Elizabeth Bannister ${ }^{d, g} \quad$ Anthea Greenway ${ }^{c}$ Natasha Brown ${ }^{\text {a, g }}$ \\ aVictorian Clinical Genetics Services, Murdoch Children's Research Institute, Melbourne, Australia; \\ bSchool of Medicine, Griffith University, Gold Coast, Australia; 'Department of Haematology, Royal Children's \\ Hospital, Melbourne, Australia; ${ }^{\mathrm{d}}$ Department of Gastroenterology, Royal Children's Hospital, Melbourne, Australia; \\ e Department of Clinical Pathology, University of Melbourne, Melbourne, Australia; ${ }^{\mathrm{f} A u s t r a l i a n}$ Genomics \\ Health Alliance, Parkville, Australia; ${ }^{9}$ Department of Paediatrics, University of Melbourne, Melbourne, Australia; \\ h'Department of Neonatal Medicine, Royal Children's Hospital, Melbourne, Australia
}

\section{Established Facts}

- Hereditary spherocytosis (HS) is the most common cause of neonatal nonimmune hemolytic anemia and confers considerable morbidity and mortality.

- Management of severe neonatal-onset HS emphasizes aggressive transfusion and prevention of hyperbilirubinemia.

- Heterozygous pathogenic variants in SPTB cause autosomal dominant $\beta$-spectrin deficient HS.

- Biallelic mutations are rarely reported.

- Rapid whole-exome sequencing has demonstrated feasibility and cost-effectiveness in acute care settings.

\section{Novel Insights}

- A novel biallelic SPTB variant (c.6119C>T) causes severe neonatal-onset HS.

- This variant in homozygous states confers severe transfusion-dependent hemolytic anemia, conjugated hyperbilirubinemia, and progressive liver failure.

- Asymptomatic heterozygotes demonstrate mild hematologic manifestations.

- Early rapid molecular diagnosis facilitates hypertransfusion to suppress ineffective erythropoiesis and reverse hepatic dysfunction.

- Rapid whole-exome sequencing should be considered in the critically ill neonate with nonimmune hemolytic anemia.

\section{Keywords}

Hereditary spherocytosis - Medical genomics - Rapid exome sequencing $\cdot$ Red cell membrane defects $\cdot$ Red cells

\begin{abstract}
Heterozygous pathogenic variants in SPTB cause autosomal dominant hereditary spherocytosis, an important cause of neonatal nonimmune hemolytic anemia. Biallelic mutations
\end{abstract}

\section{KARGER}

(C) 2020 S. Karger AG, Basel

karger@karger.com

www.karger.com/msy
Dr. Natasha Brown

Victorian Clinical Genetics Services, Murdoch Children's Research Centre Flemington Rd.

Parkville, VIC 3052 (Australia)

E-Mail natasha.brown@vcgs.org.au 
are rarely reported, all with severe neonatal presentation. We describe rapid ( $68 \mathrm{~h}$ ) genomic diagnosis of homozygous $\beta$-spectrin deficiency in a newborn with severe transfusiondependent hemolytic anemia, conjugated hyperbilirubinemia, and progressive liver failure. Trio whole-exome sequencing identified a novel biallelic SPTB variant (c.6119C > T; p.Thr2040lle) located in the critical spectrin repeat region. Pretransfusion blood film showed marked spherocytosis including microspherocytes and nucleated erythrocytes, and eosin-5-maleimide (E5M) staining was markedly reduced, supporting pathogenicity. Both asymptomatic heterozygous parents demonstrated mildly reduced E5M staining, with occasional spherocytes and elliptocytes. Early molecular diagnosis facilitated hypertransfusion to suppress ineffective erythropoiesis and reverse hepatic dysfunction. This report broadens the genotypic and phenotypic spectrum of spectrin deficiency and highlights the utility of rapid genomic testing in facilitating early diagnosis and informing targeted therapy in critically ill patients.

(c) 2020 S. Karger AG, Basel

Hereditary spherocytosis (HS) is the most common cause of congenital nonimmune hemolytic anemia in Northern European populations and is a source of considerable morbidity and mortality across the lifespan [Perrotta et al., 2008]. Genetic and clinical heterogeneity is recognized with presentations ranging from fatal prenatal hydrops [Whitfield et al., 1991; Gallagher et al., 1995] to late diagnosis in adulthood [Friedman et al., 1988]. Defects in several membrane components are implicated, including $\alpha$ - and $\beta$-spectrin (encoded by SPTA 1 and $S P T B$, respectively), ankyrin (ANK1), and less commonly band 3 (SLC4A1) and protein 4.2 (EPB42). HS results from deficiency and destabilization of the erythrocyte cell membrane, leading to reduced deformability and selective retention and destruction in the spleen [Perrotta et al., 2008]. Monoallelic dominant-acting mutations in SPTB account for $15-30 \%$ of the HS cases in Northern Europeans [Perrotta et al., 2008]. Biallelic mutations have been infrequently reported, all with severe neonatal phenotype [Tse et al., 1989; Sahr et al., 1993; Gallagher et al., 1995, 1997] (Table 1). In these reports, clinically asymptomatic heterozygotes often had subtle blood film abnormalities.

Management of severe neonatal-onset HS emphasizes aggressive transfusion and prevention of hyperbilirubinemia and associated comorbidities, often requiring multidisciplinary support in neonatal intensive care. Early molecular diagnosis permits timely, targeted therapy, aids prognostication, and may avoid multiple investigations. Utilization of next-generation sequencing in infants with conjugated hyperbilirubinemia and anemia has diagnostic utility and is cost-effective [Agarwal et al., 2016]. Rapid genomic sequencing has demonstrated feasibility and cost-effectiveness in acute care settings [Farnaes et al., 2018; Stark et al., 2018]. Molecular characterization and rationalized treatment can now be achieved in days rather than months. We describe rapid identification of a novel biallelic SPTB mutation in a newborn with severe congenital hemolytic anemia and progressive liver dysfunction.

\section{Case Report}

The female proband was born following an uncomplicated term pregnancy to healthy consanguineous Iraqi parents (Fig. 1A). Presenting features are summarized in Table 1. At 3 hours of life, she developed hypoglycemia, hypothermia and hypoxia, with a hemoglobin (Hb) concentration of $57 \mathrm{~g} / \mathrm{L}$ (reference 105-135 g/L) and raised serum lactate. Following initial resuscitation and erythrocyte transfusion, there was persistent anemia $(\mathrm{Hb} 83 \mathrm{~g} / \mathrm{L})$, and she developed hepatomegaly with significant conjugated hyperbilirubinemia, coagulopathy, and hyperferritinemia.

Early investigations targeted a broad differential diagnosis, including congenital infection, storage disorders, gestational alloimmune liver disease, hemophagocytic lymphohistiocytosis, and organic acidemias. All results were nondiagnostic. Liver biopsy was not performed due to coagulopathy and clinical instability. Blood film (Fig. 1B) identified microcytic anemia and reticulocytosis with increased polychromasia, spherocytes and nucleated red cells, compatible with HS. A primary erythrocyte membrane defect was considered and genomic testing initiated on day 3 of life. The severity of the presentation directed rapid WES with concurrent mitochondrial whole-genome sequencing to exclude a mitochondrial condition.

The infant was recruited to the Australian Genomics Acute Care Genomics study, which offers rapid trio WES +/- tandem mitochondrial whole-genome sequencing to pediatric patients in intensive care with suspected monogenic conditions. Result return is typically less than 5 days.

\section{Materials and Methods}

DNA was extracted from peripheral blood, from infant and both parents, using QIAamp Blood Mini Kits (Qiagen, Düsseldorf, Germany). Sample quality was confirmed using the Agilent Tape Station Genomic DNA ScreenTape (Agilent Technologies, Santa Clara, CA, USA). Exome sequencing was performed at a National Association of Testing Authorities-accredited laboratory (Victorian Clinical Genetics Services, Melbourne, Australia) by massively-parallel sequencing using SureSelect QXT CRE exome capture (Agilent) on a NextSeq 500 sequencer (Illumina, San Diego, CA, USA). Targeted mean coverage was $100 \times$ (minimum $90 \%$ of bases sequenced to at least $15 \times$ ). The DRAGENTM (Dynamic Read 
Table 1. Previously reported individuals with homozygous SPTB variants and associated clinical findings

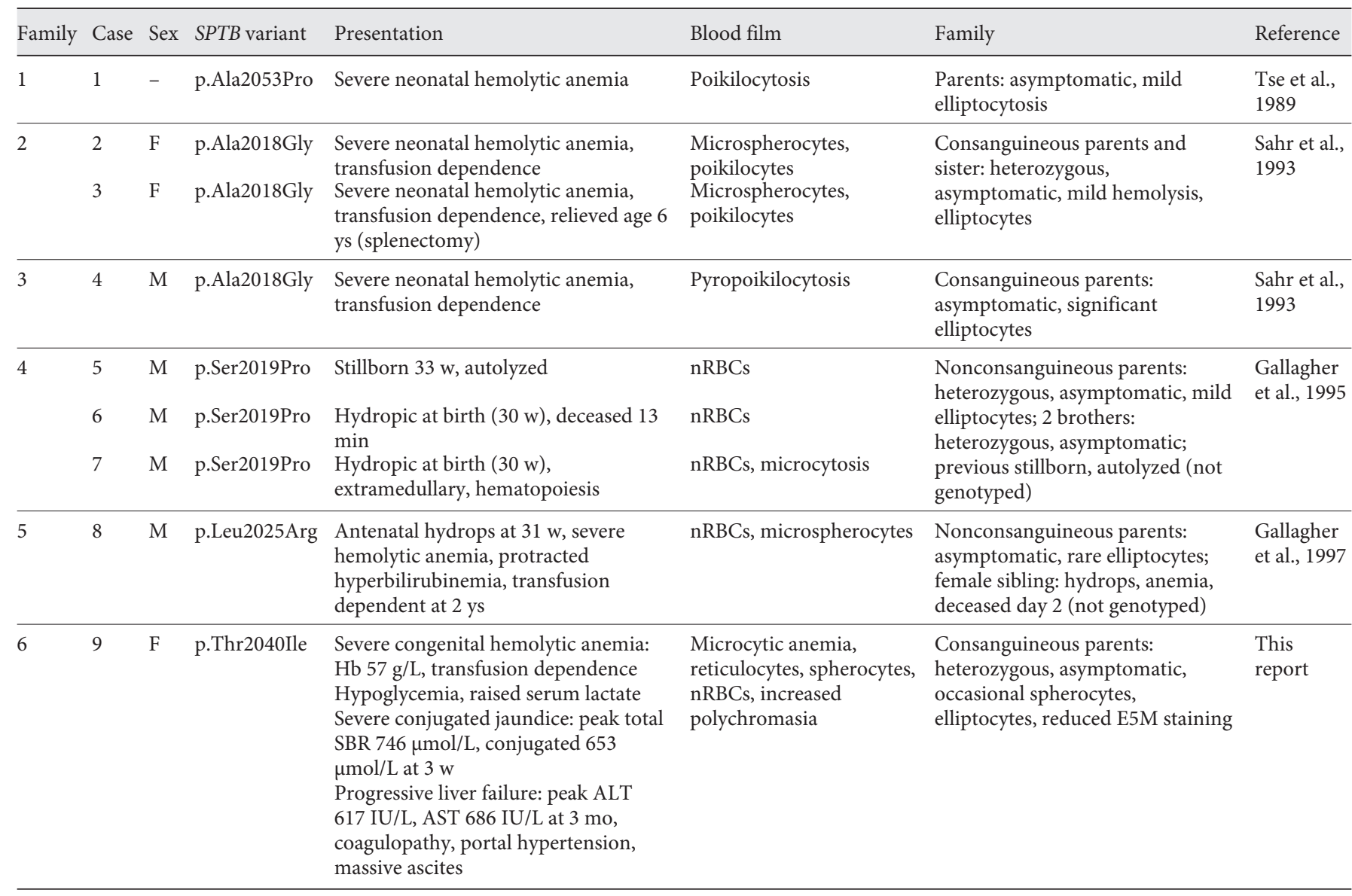

ALT, alanine aminotransferase [reference range 12-37 IU/L]; AST, aspartate aminotransferase [reference range 20-80 IU/L]; Hb, hemoglobin [reference range $105-135 \mathrm{~g} / \mathrm{L}$ ]; nRBCs, nucleated red blood cells; SBR, serum bilirubin [reference range $0-15 \mu \mathrm{mol} / \mathrm{L}$ total, $0-5 \mu \mathrm{mol} / \mathrm{L}$ conjugated]; w, weeks; ys, years.

Analysis for GENomics) Bio-IT Platform (Illumina) generated annotated single nucleotide and insertion/deletion variant calls within the target region via alignment to reference genome GRCh37. The whole mitochondrial genome was amplified using single longrange PCR followed by library preparation using the Nextera XT DNA kit (Illumina). Sequencing was performed on a MiSeq using v2 chemistry (Illumina). Data processing was undertaken using the on-board MiSeq Reporter for variant calling and a custom inhouse analysis pipeline for annotation and insertion/deletion calling. Variant prioritization was phenotype-driven and conducted by a multidisciplinary team of clinical geneticists and genomic scientists. Variants were classified according to the American College of Medical Genetics and Genomics classification criteria [Richards et al., 2015].

WES identified a novel homozygous missense variant in exon 29 of SPTB (NM_001024858.3(SPTB):c.6119C $>$ T) in the proband, with predicted moderate amino acid change at position 2040 (p.Thr2040Ile); both parents were heterozygous. This residue is moderately conserved in 100 vertebrates [Kent et al., 2002] and located within the critical spectrin repeat region in which the ma- jority of previously reported pathogenic missense variants occur [PM1] [Gallagher et al., 1997]. In silico predictions were consistently pathogenic [PP3] (PolyPhen-2 [Adzhubei et al., 2010], SIFT [Kumar et al., 2009], and MutationTaster2 [Schwarz et al., 2014]). This variant has not previously been reported in clinical cases and is absent in population databases [PM2] (gnomAD [Karczewski et al., 2019], 1000 Genomes [1000 Genomes Project Consortium et al., 2015]). Spectrin deficiency was considered a strong and specific match for the proband's phenotype [PP4], and the variant was classified as likely pathogenic. Mitochondrial whole-genome sequencing did not identify any variants of interest.

Additional functional evidence was considered. Pre-transfusion eosin-5-maleimide (E5M) flow cytometry demonstrated markedly reduced staining (ratio $<0.6$ compared to control) supporting a primary spectrin defect and variant pathogenicity (Fig. 1C). E5M staining in both parents was mildly reduced, with occasional spherocytes in the maternal blood film and elliptocytes in the paternal film. A molecular diagnosis of $\beta$-spectrin deficiency was made on day 5 . Time from sample receipt to WES result was $68 \mathrm{~h}$.
Richmond/Campbell/Foo/Lunke/Stark/ Moody/Bannister/Greenway/Brown 


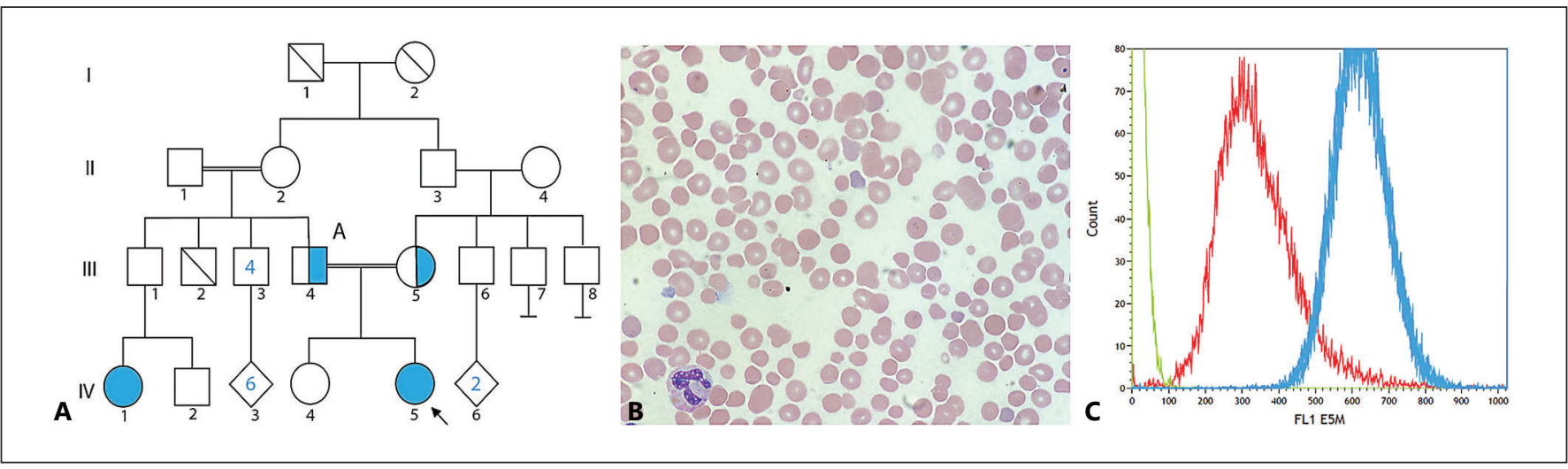

Fig. 1. Clinical and laboratory data supporting pathogenicity of the $S P T B$ variant. A Family pedigree. The blue circle indicates hereditary spherocytosis. IV:5 Proband (homozygous for the SPTB variant). II:1, II:2 are second cousins. III:4, III:5 are first cousins and both heterozygous for the SPTB variant. III: 2 deceased at 6 months due to unknown cause. IV:1 had a clinical diagnosis of HS, and was not available for genotyping. B Photomicrograph of proband blood film demonstrating microcytic anemia with the presence of reticulocytes, spherocytes, and nucleated red cells, suggestive of hemolysis. Images were captured at room temperature $\left(24^{\circ} \mathrm{C}\right)$ by Olympus (Tokyo, Japan) BX41 microscopy with an Apple (CA, USA) iPhone 8 camera following hematoxylin-and-eosin staining. Original magnification $x 100$. C Pre-transfusion eosin-5-maleimide flow cytometry in the proband demonstrated markedly reduced staining compared to control (ratio $<0.6$ suggesting an erythrocyte membrane disorder).

\section{Results}

The infant remained transfusion dependent with marked hemolysis, icterus, and evolving massive hepatosplenomegaly. Severe hepatic dysfunction was evidenced by ongoing conjugated hyperbilirubinemia (total and conjugated serum bilirubin 746 and $653 \mu \mathrm{mol} / \mathrm{L}$ respectively, peaking at 3 weeks), transaminitis (peak ALT 617 IU/L, AST $686 \mathrm{IU} / \mathrm{L}$ at 3 months), and synthetic dysfunction (coagulopathy, hypoalbuminemia; Table 1). Progressive portal hypertension and massive ascites were present by 3 months. Due to the severe conjugated hyperbilirubinemia, alternative diagnoses were investigated. Urinary bile acids were increased on mass spectrometry, consistent with cholestasis. Alpha-1-antitrypsin levels (Pi-M phenotype) and infective hepatitis screens were normal. Reanalysis of WES data did not identify a second monogenic disorder to explain the liver disease. A chromosomal microarray did not identify any copy number variants of clinical significance.

Hypertransfusion was initiated at 3 months to suppress ineffective endogenous erythropoiesis. This consisted of erythrocyte transfusions initially every 1-3 days to maintain a target hemoglobin concentration of 140 $150 \mathrm{~g} / \mathrm{L}$. Additional treatments included oral hydroxyurea, ursodeoxycholic acid, folic acid and vitamin supplementation, and nutritional support. Management of ascites required albumin infusions, diuretics, and emergency peritoneal paracentesis for respiratory compromise. Stabilization and some reversibility of hepatic dysfunction were achieved. Chronic hemolysis remained evident at 6 months (normalized total unconjugated bilirubin; slightly elevated $\gamma$-glutamyl transferase). Hepatic biopsy showed hepatic siderosis, and chelation therapy was initiated for iron overload. Growth and development were age appropriate at 6 months.

\section{Discussion and Conclusion}

The critically unwell neonate with hemolytic anemia poses diagnostic and management challenges. Diagnosis of an erythrocyte membrane defect often requires multiple investigations, including peripheral blood film, specialized staining and flow cytometry, and exclusion of other conditions. Reduced fluorescence on E5M flow cytometry supports the diagnosis of HS [Christensen et al., 2015], especially with accompanying blood film findings of hemolysis and spherocytosis, but can also occur in other conditions such as congenital dyserythropoietic anemia. Rapid genomic sequencing may transform these investigative pathways. Prompt diagnosis facilitates early correction of anemia and prevention of comorbidities, including hyperbilirubinemia, and hypersplenism. We describe a critically ill neonate with homozygous SPTB mutation causing severe hepatic dysfunction. Review of 
the literature revealed 5 previously reported families with confirmed homozygous SPTB mutations, all with severe congenital hemolytic anemia [Tse et al., 1989; Sahr et al., 1993; Gallagher et al., 1995, 1997].

In individuals with $\beta$-spectrin deficiency, including biallelic reports, severe hepatic dysfunction is uncommon [Christensen et al., 2014; Nussenzveig et al., 2014], prompting consideration of a blended phenotype in our case. Additional causes of hepatic dysfunction were excluded, including alloimmune hemolysis [Sivan et al., 1983; Ree et al., 2017], pyruvate kinase deficiency [Raphael et al., 2007], intrahepatic cholestasis [Wree et al., 2011], and hemochromatosis [Hoblinger et al., 2009]. WES did not identify a second monogenic disorder to explain the liver disease. We concluded that this infant's $\beta$-spectrin deficiency was the cause of the liver dysfunction, extending the phenotypic spectrum of HS. The exact pathogenesis of progressive liver failure is unclear; however, intrahepatic extramedullary hematopoiesis, caused by anemia and hemolysis, may contribute [Sivan et al., 1983].

Rapid genomic testing permitted diagnosis of a $\beta$-spectrin deficiency on day 5 of life, the earliest reported molecular diagnosis of HS. Confirmation of the novel homozygous c.6119C $>\mathrm{T}$ variant broadens the genotypic and phenotypic spectrum of HS caused by $\beta$-spectrin deficiency. Minor hematological anomalies in the asymptomatic heterozygous parents suggests that otherwise healthy carriers of biallelic HS may have subclinical manifestations. Early diagnosis permitted diagnostic confidence and initiation of targeted therapy, with hypertransfusion reversing the conjugated hyperbilirubinemia and hepatic dysfunction by 6 months. Family benefits of early diagnosis include diagnostic clarity during an emotionally challenging time and informed future reproductive planning.

This case demonstrates the feasibility of rapid trio WES in neonates with severe hemolytic anemia, highlighting the clinical and social utility of an early rapid diagnosis. Reanalysis of exome data with new phenotypic information provided reassurance that a secondary diagnosis had not been missed. Where available, rapid genomic testing should be considered in the diagnostic pathway for critically ill neonates with nonimmune hemolytic anemia.

\section{Acknowledgments}

The authors wish to acknowledge the families who have participated in the Australian Genomics Acute Care flagship and, in particular, the family of this patient for sharing their experience with us. We also acknowledge and value the contributions of clinical and laboratory personnel involved in the care and diagnosis of this patient.

\section{Statement of Ethics}

The Australian Genomics Acute Care Genomics project has Human Research Ethics Committee approval (HREC/16/MH251), and informed consent was obtained from the family for genomic testing and case report publication, in accordance with institutional requirements.

\section{Disclosure Statement}

The authors have no conflicts of interest to declare.

\section{Funding Sources}

The Australian Genomics Acute Care study is funded by the Australian National Health and Medical Research Council Targeted Call for Research (GNT1113531) and the Royal Children's Hospital Foundation (2017-906).

\section{Author Contributions}

Christopher Richmond, Sally Campbell, Hee Wei Foo, Amanda Moody, Elizabeth Bannister, Anthea Greenway, and Natasha Brown provided clinical care to the patient and family and prepared clinical details for the paper. Sebastian Lunke and Zornitza Stark are project leads for the Australian Genomics Acute Care Genomics project. Christopher Richmond, Sebastian Lunke, Zornitza Stark, and Natasha Brown analyzed results. Sally Campbell prepared images. Christopher Richmond wrote the paper, and Sebastian Lunke, Zornitza Stark, Elizabeth Bannister, Anthea Greenway, and Natasha Brown provided senior clinical and editorial oversight.

References
Adzhubei IA, Schmidt S, Peshkin L, Ramensky
VE, Gerasimova A, et al: A method and server
for predicting damaging missense mutations.
Nat Methods 7:248-249 (2010).
Agarwal AM, Nussenzveig RH, Reading NS, Patel
JL, Sangle N, et al: Clinical utility of next-gen-
eration sequencing in the diagnosis of heredi-
tary hemolytic anemias. Br J Haematol 174:
806-814 (2016).
Christensen RD, Nussenzveig RH, Yaish HM,
Henry E, Eggert LD, Agarwal AM: Causes of
hemolysis in neonates with extreme hyperbil-
irubinemia. J Perinatol 34:616-619 (2014).
Christensen RD, Agarwal AM, Nussenzveig RH,
Heikal N, Liew MA, Yaish HM: Evaluating
eosin-5-maleimide binding as a diagnostic
test for hereditary spherocytosis in newborn
infants. J Perinatol 35:357-361 (2015).

Richmond/Campbell/Foo/Lunke/Stark/ Moody/Bannister/Greenway/Brown 
Farnaes L, Hildreth A, Sweeney NM, Clark MM, Chowdhury S, et al: Rapid whole-genome sequencing decreases infant morbidity and cost of hospitalization. NPJ Genom Med 3:10 (2018).

Friedman EW, Williams JC, Van Hook L: Hereditary spherocytosis in the elderly. Am J Med 84:513-516 (1988)

-Gallagher PG, Weed SA, Tse WT, Benoit L, Morrow JS, et al: Recurrent fatal hydrops fetalis associated with a nucleotide substitution in the erythrocyte beta-spectrin gene. J Clin Invest 95:1174-1182 (1995).

-Gallagher PG, Petruzzi MJ, Weed SA, Zhang Z, Marchesi SL, et al: Mutation of a highly conserved residue of betaI spectrin associated with fatal and near-fatal neonatal hemolytic anemia. J Clin Invest 99:267-277 (1997).

1000 Genomes Project Consortium, Auton A, Brooks LD, Durbin RM, Garrison EP, et al: A global reference for human genetic variation. Nature 526:68-74 (2015).

-Hoblinger A, Erdmann C, Strassburg CP, Sauerbruch T, Lammert F: Coinheritance of hereditary spherocytosis and reversibility of cirrhosis in a young female patient with hereditary hemochromatosis. Eur J Med Res 14:182-184 (2009).

Karczewski KJ, Francioli LC, Tiao G, Cummings BB, Alföldi J, et al: Variation across 141,456 human exomes and genomes reveals the spectrum of loss-of-function intolerance across human protein-coding genes. bioRxiv:531210 (2019).
Kent WJ, Sugnet CW, Furey TS, Roskin KM, Pringle TH, et al: The human genome browser at UCSC. Genome Res 12:996-1006 (2002).

Kumar P, Henikoff S, Ng PC: Predicting the effects of coding non-synonymous variants on protein function using the SIFT algorithm. Nat Protoc 4:1073-1081 (2009).

- Nussenzveig RH, Christensen RD, Prchal JT, Yaish HM, Agarwal AM: Novel alpha-spectrin mutation in trans with alpha-spectrin causing severe neonatal jaundice from hereditary spherocytosis. Neonatology 106:355357 (2014).

errotta S, Gallagher PG, Mohandas N: Hereditary spherocytosis. Lancet 372:1411-1426 (2008).

Raphael MF, Van Wijk R, Schweizer JJ, Schoutenvan Meeteren NA, Kindermann A, et al: Pyruvate kinase deficiency associated with severe liver dysfunction in the newborn. Am J Hematol 82:1025-1028 (2007).

Ree IMC, Smits-Wintjens V, van der Bom JG, van Klink JMM, Oepkes D, Lopriore E: Neonatal management and outcome in alloimmune hemolytic disease. Expert Rev Hematol 10:607616 (2017)

Richards S, Aziz N, Bale S, Bick D, Das S, et al: Standards and guidelines for the interpretation of sequence variants: a joint consensus recommendation of the American College of Medical Genetics and Genomics and the Association for Molecular Pathology. Genet Med 17:405-424 (2015).
Sahr KE, Coetzer TL, Moy LS, Derick LH, Chishti $\mathrm{AH}$, et al: Spectrin cagliari. An Ala $\rightarrow$ Gly substitution in helix 1 of beta spectrin repeat 17 that severely disrupts the structure and selfassociation of the erythrocyte spectrin heterodimer. J Biol Chem 268:22656-22662 (1993).

Schwarz JM, Cooper DN, Schuelke M, Seelow D: MutationTaster2: mutation prediction for the deep-sequencing age. Nat Methods 11:361362 (2014).

Sivan Y, Merlob P, Nutman J, Reisner SH: Direct hyperbilirubinemia complicating $\mathrm{ABO}$ hemolytic disease of the newborn. Clin Pediatr (Phila) 22:537-538 (1983).

-Stark Z, Lunke S, Brett GR, Tan NB, Stapleton R, et al: Meeting the challenges of implementing rapid genomic testing in acute pediatric care. Genet Med 20:1554-1563 (2018).

Tse W, Costa F, Lecomte M, Dhermy D, Garbarz $M$, et al: An Ala-to-Pro substitution in the $\beta$-spectrin chain causes $\alpha^{\mathrm{I} / 74}$ hereditary elliptocytosis (HE). Blood 74 (Suppl 1):105a (1989).

Whitfield CF, Follweiler JB, Lopresti-Morrow L, Miller BA: Deficiency of alpha-spectrin synthesis in burst-forming units-erythroid in lethal hereditary spherocytosis. Blood 78:30433051 (1991).

Wree A, Canbay A, Müller-Beissenhirtz H, Dechêne A, Gerken G, et al: Excessive bilirubin elevation in a patient with hereditary spherocytosis and intrahepatic cholestasis. Z Gastroenterol 49:977-980 (2011). 